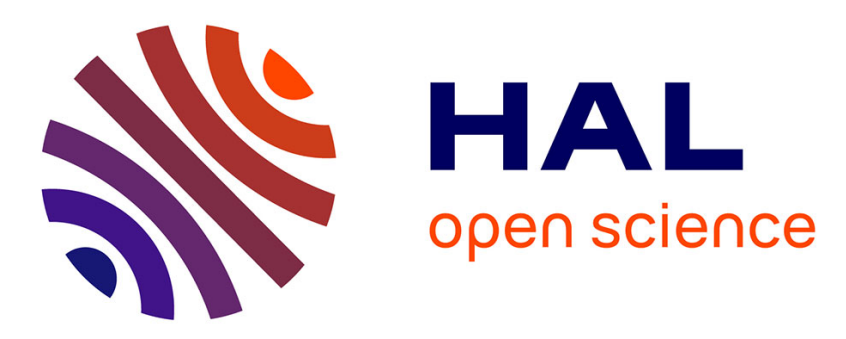

\title{
Numerical investigation of BB-AMR scheme using entropy production as refinement criterion
}

Thomas Altazin, Mehmet Ersoy, Frederic Golay, Damien Sous, Lyudmyla Yushchenko

\section{- To cite this version:}

Thomas Altazin, Mehmet Ersoy, Frederic Golay, Damien Sous, Lyudmyla Yushchenko. Numerical investigation of BB-AMR scheme using entropy production as refinement criterion. International Journal of Computational Fluid Dynamics, 2016, 10.1080/10618562.2016.1194977 . hal-01330654

\section{HAL Id: hal-01330654 https://hal.science/hal-01330654}

Submitted on 8 Jul 2016

HAL is a multi-disciplinary open access archive for the deposit and dissemination of scientific research documents, whether they are published or not. The documents may come from teaching and research institutions in France or abroad, or from public or private research centers.
L'archive ouverte pluridisciplinaire $\mathbf{H A L}$, est destinée au dépôt et à la diffusion de documents scientifiques de niveau recherche, publiés ou non, émanant des établissements d'enseignement et de recherche français ou étrangers, des laboratoires publics ou privés. 


\title{
Numerical investigation of BB-AMR scheme using entropy production as refinement criterion
}

\author{
Thomas Altazin*1 $^{* 1}$, Mehmet Ersoy ${ }^{\dagger 1}$, Frédéric Golay ${ }^{\ddagger 1}$, Damien Sous ${ }^{\S 2}$, and Lyudmyla \\ Yushchenko 11 \\ ${ }^{1}$ Université de Toulon, IMATH, EA 2134, 83957 La Garde, France. \\ ${ }^{2}$ Université de Toulon, Aix-Marseille Université, CNRS/INSU, IRD, MIO, UM 110, 13288, \\ Marseille, Cedex 09, France.
}

September 2, 2015

\begin{abstract}
In this work, a parallel finite volume scheme on unstructured meshes is applied to fluid flow for multidimensional hyperbolic system of conservation laws. It is based on a block-based adaptive mesh refinement strategy which allows quick meshing and easy parallelisation. As a continuation and as an extension of a previous work, the useful numerical density of entropy production is used as mesh refinement criterion combined with a local time stepping method to preserve the computational time. Then, we numerically investigate its efficiency through several test cases with a confrontation with exact solution or experimental data.
\end{abstract}

Keywords: multidimensional hyperbolic system, finite volume, adaptive mesh refinement, numerical density of entropy production, local time stepping

\section{Introduction}

The numerical simulation of two-phases fluid flows in complex three-dimensional configurations remains a challenging task constrained by two conflicting goals: the precise description of each involved physical processes over the whole simulated domain down to the smallest spatio-temporal scales of the fluid motion and the computational cost. In reality, a compromise is generally made according to the requirements by the studied physical cases (for a more detailed presentation of the numerical/physical strategic issues of such compromise in the particular case of wave impact on rigid structures, the reader can refer, for instance, to Golay et al. [10]). Our research work focuses on a multi dimensional numerical scheme able to accurately solve non-linear hyperbolic systems of conservation laws while preserving the computational time. This scheme has been first presented by Ersoy et al. [6] in the one dimensional case and further extended to three dimensions and confronted to experimental data by Golay et al. [10].

The present research work is dedicated to the study of the numerical performance of the model through several test cases. The model framework and the related scientific issues having been detailed in recent above-mentioned publications, we briefly recall here the overall principles of the Block-Based Adaptative

\footnotetext{
*Thomas.Altazin@univ-tln.fr

${ }^{\dagger}$ Mehmet.Ersoy@univ-tln.fr

${ }^{\ddagger}$ Frederic.Golay@univ-tln.fr

$\S$ damien.sous@mio.osupytheas.fr

『Lyudmyla.Yushchenko@univ-tln.fr
} 
Mesh Refinement (BB-AMR) scheme while the main numerical features of the model are presented in the next section. The equations system of interest is

$$
\begin{cases}\frac{\partial \boldsymbol{w}(t)}{\partial t}+\nabla \cdot \boldsymbol{f}(t, \boldsymbol{w})=\boldsymbol{G}, & (t, x) \in \mathbb{R}^{+} \times \mathbb{R}^{d} \\ \boldsymbol{w}(0, x)=\boldsymbol{w}_{0}(x), & x \in \mathbb{R}^{d} .\end{cases}
$$

where $\boldsymbol{w}, \boldsymbol{f}, \boldsymbol{G}$ stands respectively for conservative variables, flux and source.

As a well-known result, the uniqueness of the (weak) solution is lost even if the initial data are smooth. It can recovered by completing System (1) with an entropy inequality of the form:

$$
S=\frac{\partial s(\boldsymbol{w})}{\partial t}+\nabla \cdot \boldsymbol{\psi}(\boldsymbol{w}) \leq 0
$$

where $(s, \psi)$ stands for a convex entropy-entropy flux pair. Even if we are not able to prove the uniqueness in the multidimensional case, this inequality allows to select the physical relevant solution and provide a "smoothness" indicator since the entropy satisfies a conservation equation only in regions where the solution is smooth and an inequality when the solution develops discontinuities. Thus, the discrete quantity $S$ can always be considered as a measure of the amount of violation of the entropy equation (as pointed out in $[4,14,17,16,6])$. As already done in $[6], S$, which is called the numerical density of entropy production, can be used as a smoothness indicator providing information on the need to locally refine the mesh (e.g. if the solution develops discontinuities) or to coarsen the mesh (e.g. if the solution is smooth and wellapproximated).

A major issue of many modelling challenges is to accurately simulate processes over very large ranges in spatial scale inexorably leading to heavy computational time. Thus, in order to efficiently implement the local time stepping method in a parallel framework, the approach taken here is to equip our adaptive mesh refinement (AMR) technique with a block-based (BB-AMR) strategy. Sufficient spatio-temporal resolution can be reached in a reasonable CPU time, which is especially useful for hyperbolic problems generally requiring fine CPU-consuming uniform mesh. Roughly speaking, the BB-AMR technique provides an efficient control of the shared memory leading to well-balanced computational time between cores by domain-like decomposition. Nevertheless, data locality is critical to obtain good performance since the memory access times are not uniform and may become expensive. Consequently, the re-meshing step has to be carefuly managed. Taking advantage of such a block-based structure (as in domain decomposition), we can define two different time-step: the first one is based on the CFL condition (i.e computed through the finest cells) while the second one is defined at the level of the block. The stability of the scheme is thus respected for any time and the re-meshing cost is minimized.

The first part of this paper (section 2) is dedicated to the summarized presentation of the multidimensional finite volume solver on unstructured meshes with a Block-Based Adaptive Mesh Refinement technique (BBAMR). The second and main part of the paper (section 3) consists in an evaluation of the method performance through a series of test cases. First, as an addendum to [6], our one dimensional solver is tested on the wellknown Woodward and Colella interacting blast-wave problem [32, 31]). Additional tests are performed on classical two dimensional Riemann problem for polytropic Euler equations which have been extensively studied [35, 27, 21, 20, 22]. Solutions are composed of nineteen possible geometric configurations connected by shocks, rarefactions and contacts (see for instance [21]). The so-called configuration 17 is used here. It consists of a solution composed by a shock, a rarefaction wave and two contact discontinuities. The final set of numerical tests concerns the 2D and 3D simulation of two-phases flows on a dam-break problem. For that cases, our model is based on a three-dimensional compressible low Mach two-phase flows model with a linearized "artificial pressure" law which physical relevancy has already been demonstrated in the context of highly dynamical and aerated flows for breaking and impacting waves by Sambe et al. [26] or Golay et al. [10]. 


\section{Finite volume approximation for hyperbolic conservation laws}

This section summarizes the main features of our method, including the semi-discrete finite volume numerical approximation of a general non-linear hyperbolic equation (eq. (1)), the time integration and mesh refinement procedure. Note that $\boldsymbol{w}, \boldsymbol{f}, \boldsymbol{G}$ are the conservative variables, the flux and the source term, respectively, and $d \in \llbracket 1,3 \rrbracket$.

\subsection{Multidimensional finite volume approximation}

The computational domain $\Omega \subset \mathbb{R}^{d}$ is split into a set of control volumes, also referred as cells, $\Omega=\cup_{k} C_{k}$ of mesh size $\left|C_{k}\right|$. For the sake of simplicity the source term is here omitted.

On a given cell $C_{k}$, noting $w_{k}(t)$

$$
\boldsymbol{w}_{k}(t) \simeq \frac{1}{\left|C_{k}\right|} \int_{C_{k}} \boldsymbol{w}(t, x) d \boldsymbol{x}
$$

the approximation of the mean value of the unknown $\boldsymbol{w}(t, x)$ on $C_{k}$ at time $t$, and integrating (1) over each cell, we obtain:

$$
\int_{C_{k}} \frac{\partial \boldsymbol{w}(t)}{\partial t}+\sum_{a} \int_{\partial C_{k / a}} \boldsymbol{f}(t, \boldsymbol{w}) \cdot \boldsymbol{n}_{k / a}=0
$$

where $\boldsymbol{n}_{k / a}$ denotes the unit normal vector on the boundary $\partial C_{k / a}$ between cells $k$ and $a$.

Next, $F\left(\boldsymbol{w}_{k}(t), \boldsymbol{w}_{a}(t), \boldsymbol{n}_{k / a}\right)$ the flux approximation being written

$$
\boldsymbol{F}\left(\boldsymbol{w}_{k}(t), \boldsymbol{w}_{a}(t), \boldsymbol{n}_{k / a}\right) \approx \frac{1}{\left|\partial C_{k / a}\right|} \int_{\partial C_{k / a}} \boldsymbol{f}(t, \boldsymbol{w}) \cdot \boldsymbol{n}_{k / a} d s
$$

the semi-discrete finite volume approximation of eqs. (1) (see for instance [9, 29, 7]) are obtained:

$$
\frac{\partial \boldsymbol{w}_{k}(t)}{\partial t}+\frac{1}{\left|C_{k}\right|} \sum_{a}\left|\partial C_{k / a}\right| \boldsymbol{F}\left(\boldsymbol{w}_{k}(t), \boldsymbol{w}_{a}(t), \boldsymbol{n}_{k / a}\right)=0
$$

where $\boldsymbol{F}\left(\boldsymbol{w}_{k}(t), \boldsymbol{w}_{a}(t), \boldsymbol{n}_{k / a}\right)$ is defined via the Godunov solver, i.e. it is computed with the exact solution of the 1D Riemann problem at the interface $k / a$ with the states $w_{k}(t)$ and $w_{a}(t)$ (for further details see, for instance, [29] or [11]).

Equation (1) is completed with the entropy inequality of eq. (2) where

$$
\left(\nabla_{w} \boldsymbol{\psi}(s(\boldsymbol{w}))\right)^{t}=\left(\nabla_{w} s(\boldsymbol{w})\right)^{t} D_{\boldsymbol{w}} f(\boldsymbol{w})
$$

Following Ersoy et al. [6], this inequality is approximated using the semi-discrete finite volume scheme (4). The obtained discrete quantity, called the numerical density of entropy production, is then used as a mesh refinement criterion (see Section 2.3).

For further details on the construction of the numerical scheme we refer to [6] since the definition of the numerical fluxes reduce to a one dimensional computational at each interface $k / a$. Up to now, the first and second order Godunov schemes are implemented.

\subsection{Time integration}

The time integration of eqs. (4) and (2) can be achieved in a classical way either by a Runge-Kutta or Adams-Bashforth scheme. Note that, even if the Adams-Bashforth scheme is known to be less stable and less accurate, it can be easily handled in the framework of local time stepping to save computational time (see e.g. [2] or [6]). 


\subsubsection{Runge Kutta schemes}

By integrating eq. (4) (and (2)) during the time step $] t_{n}, t_{n+1}\left[\right.$ of length $\delta t_{n}$ and by evaluating the numerical fluxes at time $t_{n}$, the well-known first order Euler's scheme is obtained:

$$
\boldsymbol{w}_{k}\left(t_{n+1}\right)=\boldsymbol{w}_{k}\left(t_{n}\right)-\frac{\delta t_{n}}{\left|C_{k}\right|} \sum_{a}\left|\partial C_{k / a}\right| \boldsymbol{F}\left(\boldsymbol{w}_{k}\left(t_{n}\right), \boldsymbol{w}_{a}\left(t_{n}\right), \boldsymbol{n}_{k / a}\right) .
$$

In order to increase the accuracy, a second order Runge-Kutta method can be used as follows

$$
\boldsymbol{w}_{k}\left(t_{n+1}\right)=\boldsymbol{w}_{k}\left(t_{n}\right)-\frac{\delta t_{n}}{\left|C_{k}\right|} \sum_{a}\left|\partial C_{k / a}\right| \boldsymbol{F}\left(\boldsymbol{w}_{k}\left(t_{n+1 / 2}\right), \boldsymbol{w}_{a}\left(t_{n+1 / 2}\right), \boldsymbol{n}_{k / a}\right)
$$

where

$$
\boldsymbol{w}_{k}\left(t_{n+1 / 2}\right)=\boldsymbol{w}_{k}\left(t_{n}\right)-\frac{\delta t_{n}}{2\left|C_{k}\right|} \sum_{a}\left|\partial C_{k / a}\right| \boldsymbol{F}\left(\boldsymbol{w}_{k}\left(t_{n}\right), \boldsymbol{w}_{a}\left(t_{n}\right), \boldsymbol{n}_{k / a}\right) .
$$

The numerical density of entropy production (2) is then obtained with a second order Runge-Kutta scheme.

\subsubsection{Adams-Bashforth schemes}

The Adams-Bashforth method of order $m$ consists in replacing the numerical flux of Equation (4) by a polynomial interpolation of the same order [13]. For example, the second order Adams-Bashforth method reads:

$$
\begin{aligned}
& \boldsymbol{w}_{k}\left(t_{n+1}\right)=\boldsymbol{w}_{k}\left(t_{n}\right)-\frac{\delta t_{n}}{\left|C_{k}\right|} \sum_{a}\left|\partial C_{k / a}\right| \boldsymbol{F}\left(\boldsymbol{w}_{k}\left(t_{n}\right), \boldsymbol{w}_{a}\left(t_{n}\right), \boldsymbol{n}_{k / a}\right) \\
& -\frac{\delta t_{n}^{2}}{2 \delta t_{n-1}\left|C_{k}\right|}\left(\sum_{a}\left|\partial C_{k / a}\right| \boldsymbol{F}\left(\boldsymbol{w}_{k}\left(t_{n}\right), \boldsymbol{w}_{a}\left(t_{n}\right), \boldsymbol{n}_{k / a}\right)-\sum_{a}\left|\partial C_{k / a}\right| \boldsymbol{F}\left(\boldsymbol{w}_{k}\left(t_{n-1}\right), \boldsymbol{w}_{a}\left(t_{n-1}\right), \boldsymbol{n}_{k / a}\right)\right) .
\end{aligned}
$$

The Adams-Bashforth methods of order 2 and 3 are stable for Courant-Friedrich-Levy condition (CFL) less than one [1]. Practically, for stability purpose, we limit our applications to the second order scheme.

We also perform the same discretization above for entropy production (2).

\subsection{Mesh refinement criterion and BB-AMR technique}

By contrast to the one dimensional case, defining a robust mesh refinement parameter for 2 and 3D configurations is not enough to design a suitable numerical solver. The treatment of data is also a crucial point and in particular the way to spare the memory in a parallel process. This point is handled in a hierarchical block-based way that we have called BB-AMR. First, we present the main strategy to adapt the mesh with respect to the numerical density of entropy production and then how to manage data in an efficient way.

\subsubsection{Numerical entropy production as mesh refinement criterion}

The efficiency of the numerical density of entropy production as a relevant mesh refinement parameter have been already demonstrated in a previous work $[6,10]$. It has been numerically observed (and from theoretical considerations) that the production of the numerical density of entropy is almost zero for smooth solution and non-positive when the solution develops discontinuities. As a consequence, the mesh is automatically and proportionally (with respect to the production) refined inside area where the production is non zero. More precisely, Ersoy et al. [6] have demonstrated that, for the one dimensional gas dynamics equation, the support of the relative error coincides with the support of the numerical density of entropy production. The extension toward the multi dimensional case is detailed in the case of two-fluid flows in [10].

According to the finite volume approximation defined in Section 2, a local numerical entropy production $S_{k}^{n}$ is computed on each cell at time $t_{n}$ and compared to the average entropy production $\bar{S}=\frac{1}{|\Omega|} \sum_{k} S_{k}^{n}$. 
Two coefficients $0 \leqslant \alpha_{\min } \leqslant \alpha_{\max } \leqslant 1$ are thus defined to determine the ratio of numerical production of entropy leading to mesh refinement or mesh coarsening.

For each cell $C_{k}$ :

- if $S_{k}^{n}>\bar{S} \alpha_{\max }$, the mesh is refined and split and,

- if $S_{k}^{n}<\bar{S} \alpha_{\min }$ the mesh is coarsened whenever it is possible following the rule defined hereinafter.

The threshold parameters $\alpha_{\max }$ and $\alpha_{\min }$ are empirically determined, according to the simulated case requirements, to reach a relevant compromise between computational cost and accuracy. Thus $\alpha_{\min }$ and $\alpha_{\max }$ allow to set a percentage of mesh refinement and mesh coarsening with respect to the quantity $\bar{S}$. For instance, the smaller $\alpha_{\min }$ and $\alpha_{\max }$ are, the more accurate the results are at the expense of CPU time.

\subsubsection{Mesh refinement process}

For the one dimensional case, the local mesh refinement procedure is constructed following dyadic tree applied at each time step. "Macro cells" are used to be easily refined by generating hierarchical grids. Each cell can be split in two. Dyadic cells graph are thus produced, in basis 2 numbering, to allow a quick computing scan to determine the adjacent cells. For stability reasons, the mesh refinement level cannot exceed 2 between two adjacent cells. More details can be found in [6].

The three-dimensional extension of the mesh refinment procedure is a challenging task. Interesting works have been presented for 2D Cartesian grid or quad-tree [3, 33, 25, 34], octree for 3D simulations [23, 8], and anisotropic AMR [5, 12]. The extension from 1D to 3D leads to naturally octree meshing. But, the presence of a complex moving interface (composed of rarefaction, shocks and/or contacts) implies to re-mesh at each time step, which is obviously a costly process. Guided by the need to reach a relevant compromise between the contradictory aims of solution accuracy and computing speed, a Cartesian block-based mesh approach is introduced, somehow like in $[30,36]$. The computational domain is divided in several blocks, each corresponding to the initial unstructured mesh composed of hexahedral cells. These blocks are, in their turn, splitted in a Cartesian way $\left(2^{L-1} n_{x}, 2^{L-1} n_{y}, 2^{L-1} n_{z}\right)$, where $\left(n_{x}, n_{y}, n_{z}\right)$ stands for the initial block discretisation, and $L$ the level of mesh refinement. For each refined cells (or blocks), averaged values are projected on each sub-cell and fluxes are computed as simply as possible to avoid heavy computation. Then, in order to balance the CPUs load, the cells of each block are re-distributed in a fixed number of domains according to the Cuthill-McKee numbering, see figure 1. The number of domain being fixed, each domain are loaded in a given MPI process. The re-numbering and re-meshing being expensive, the mesh is finally kept constant on a time interval, called AMR time-step, given by the smallest block (rather by the smallest cell) and the maximum velocity. More details on the 3D BB-AMR are given in [10].

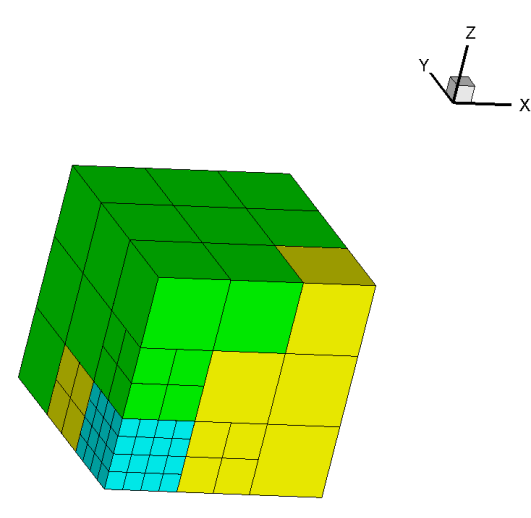

(a) Block-based mesh

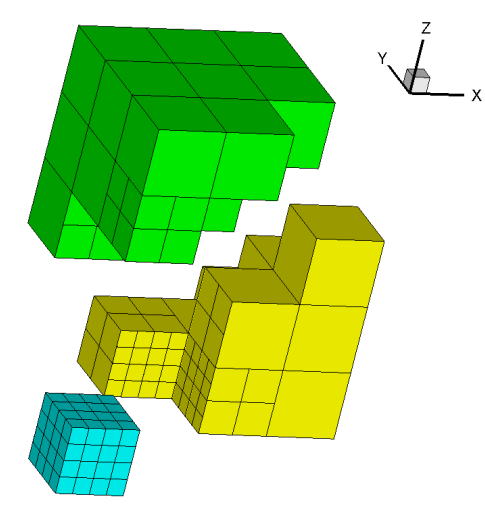

(b) Domain decomposition

Figure 1: Example of three dimensional block-based mesh with 3 domains and 27 blocks [10]. 


\section{Numerical results}

This section is dedicated to the numerical validation of the BB-AMR scheme through several multidimensional test cases.

\subsection{Euler equations of gas dynamics}

Let us consider a compressible perfect gas confined in a domain $\Omega$. The governing equations for the motion of the compressible gas in $[0, T] \times \Omega$ are the so called Euler equations

$$
\left\{\begin{aligned}
\frac{\partial \rho}{\partial t}+\nabla \cdot(\rho \boldsymbol{u}) & =0 \\
\frac{\partial \rho \boldsymbol{u}}{\partial t}+\nabla \cdot\left(\rho \boldsymbol{u} \otimes \boldsymbol{u}+p \boldsymbol{I}_{d}\right) & =0 \\
\frac{\partial \rho E}{\partial t}+\nabla \cdot((\rho E+p) \boldsymbol{u}) & =0
\end{aligned}\right.
$$

where $\rho$ is the density, $p=(\gamma-1) \rho \varepsilon$ is the pressure, $u$ the velocity and $E$ the total energy defined by:

$$
E=\varepsilon+\frac{\|\boldsymbol{u}\|^{2}}{2} .
$$

Here, $\varepsilon$ stands for the internal specific energy and $\gamma$ (set to 1.4) is the ratio between specific heats.

System (6) is written as a system of conservation laws as in eqs. (1) completed with the entropy inequality (2) where the convex entropy $s(\boldsymbol{w})=s(\rho, \rho \boldsymbol{u}, \rho E)$ and the entropy flux $\psi(\boldsymbol{w})$ are classically given by the following relations:

$$
s(\boldsymbol{w})=-\rho \ln \left(\frac{p}{\rho^{\gamma}}\right), \quad \psi(\boldsymbol{w})=u s(\boldsymbol{w}) .
$$

Let us note that even if System (6) is strictly hyperbolic on the set $\{\rho>0\}$, the previous quantities defined in eq. (7) make a sense for $\rho \geqslant 0$.

\subsubsection{One dimensional Riemann problem}

The first test case is based on the Woodward-Colella blast wave benchmark case. This one-dimensional test problem, which was initially introduced in [32], is one of the most challenging test case to solve on a uniform grid even with a very large number of cells. In particular, it illustrates the strong relationship between the accuracy of the overall flow solution and the thinness of discontinuities on the grid involving multiple interactions of discontinuities (shocks and contact discontinuities) and rarefactions.

The initial condition consists of three constant states

$$
x \in[-1,1], \quad \rho(0, x)=1, \quad u(0, x)=0, \quad p(0, x)= \begin{cases}1000, & x \leqslant 0.1 \\ 0.01, & 0.1<x \leqslant 0.9, \\ 100, & x>0.9\end{cases}
$$

on a computational domain $[0,1]$ with prescribed reflecting boundary conditions.

A full set of comparisons with different approximations is proposed here, using the following settings and notations.

- Both first and second order schemes are compared. Thus, we will refer to AB1, AB2 and RK2 as first and second order Adams-Basforth schemes and second order Runge-Kutta scheme, respectively. Both AB2 and RK2 use a MUSCL reconstruction [11]. Computations are performed on a dynamic grid with a uniform time step except:

- if the case acronym ends with " $\mathrm{U}$ ", which refers to a uniform fixed grid or, 
- if the acronym ends with "M", which refers to the local time stepping algorithm (see [6] for further details on the local time stepping adaptation).

The CPU time is noted

- $\mathrm{cpu}_{\mathrm{g}}$ for the uniform (or global) time stepping,

$-\mathrm{cpu}_{1}$ for the local time stepping.

- A reference solution (black line in fig. 2) is computed on a uniform grid with 20000 cells using the RK2U scheme and

- For all numerical tests in this section, the following parameters are used:

$\begin{array}{ll}\text { CFL } & : 0.25, \\ \text { Simulation time }(s) & : 0.038, \\ \text { Initial number of cells } & : 200, \\ \text { Maximum level of mesh refinement } & : L_{\max } . \\ \text { Mesh refinement parameter } \alpha_{\max } & : 0.01, \\ \text { Mesh coarsening parameter } \alpha_{\min } & : 0.001, \\ \text { Mesh refinement parameter } \bar{S} & : \frac{1}{|\Omega|} \sum_{k_{b}} S_{k_{b}}^{n} .\end{array}$

- Adaptive numerical solutions are compared to those computed on uniform fixed grid. For a relevant comparison, the solution on the fixed grid will be computed with $N_{L_{\max }}$ cells. $N_{L_{\max }}$ stands for the average number of cells used during a simulation of an adaptive scheme with a maximum level $L_{\max }$ which is the maximum level of refinement.

- Each presented result displays a positive density. Thus, for the sake of visual commodity, the numerical density of entropy production will be depicted in absolute value on the density plots.

- To study the numerical convergence of AB1, AB1U, AB1M, RK2U, AB2U, AB2M and RK2 cases, the discrete $l_{x}^{1}$-norm is used for density, momentum, pressure and internal energy error (which is representative of the scheme efficiency, in particular for low density flows).

- Tables of schemes performance are displayed in tabs. (1) and (2). These tables are useful to compare the computational times and, in particular, to assess the expression "saving the computational time keeping the same order of accuracy". Since the errors of the AB1 and AB1M (respectively AB2 and AB2M) schemes are similar, only the former is presented in (tab. (1)) (respectively (tab. (2))).

Figure 2 depicts the solution profiles for density (fig. 2(a), together with numerical density of entropy production $S_{k}^{n}$ ), pressure (fig. 2(b)), momentum (fig. 2(c)) and internal energy (fig. 2(d)) for the AB1U, $\mathrm{AB} 1$ and reference cases computed with $L_{\max }=5$ and 709 cells (in average for the AB1 case). Figure 2(a) first demonstrates the relevancy of the entropy production as refinement criterion: the stronger the density gradient, the larger the density of entropy production is. Each of the plotted profiles then shows the solution improvement provided by the use of adaptive mesh. 


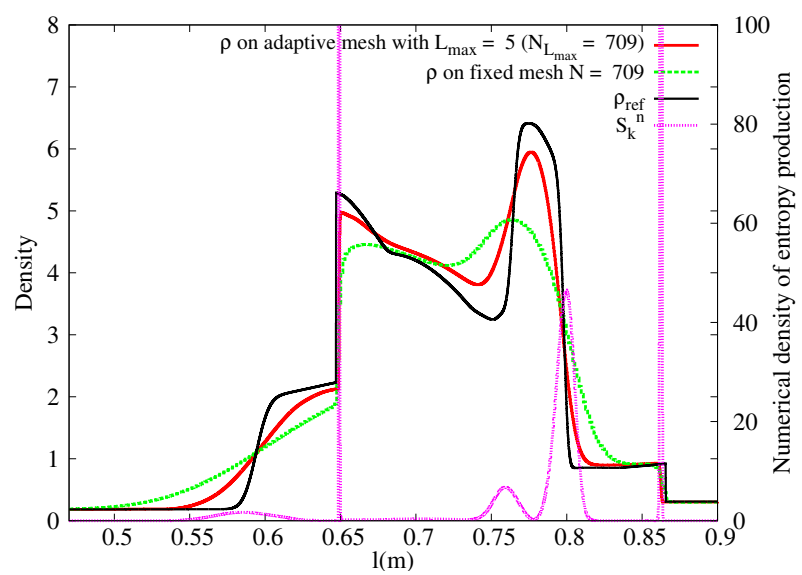

(a) Density and numerical density of entropy production.

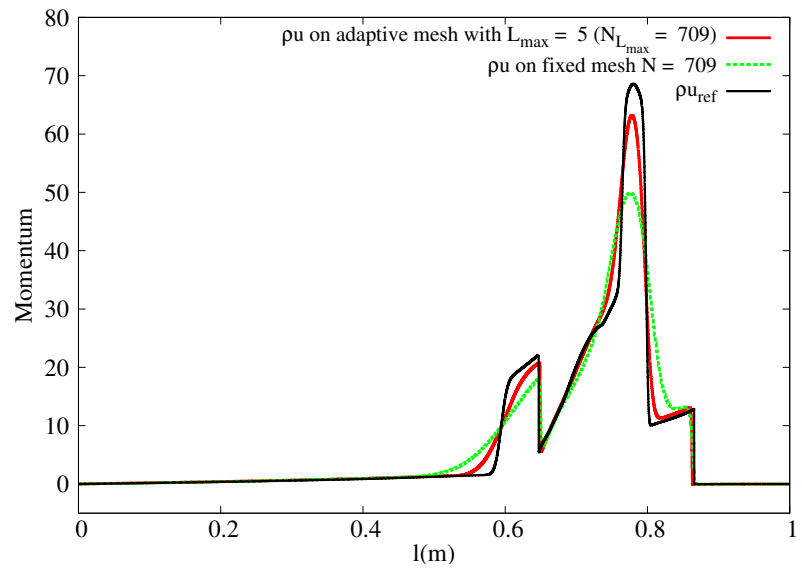

(c) Momentum.

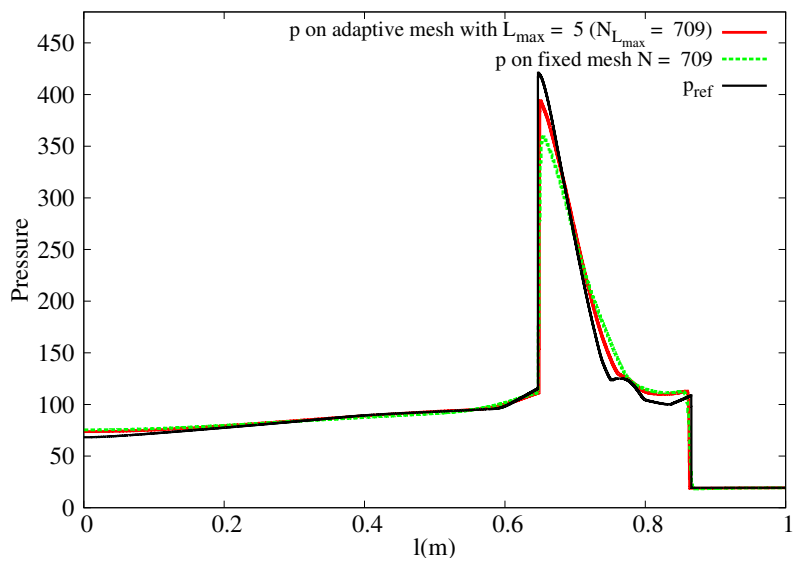

(b) Pressure.

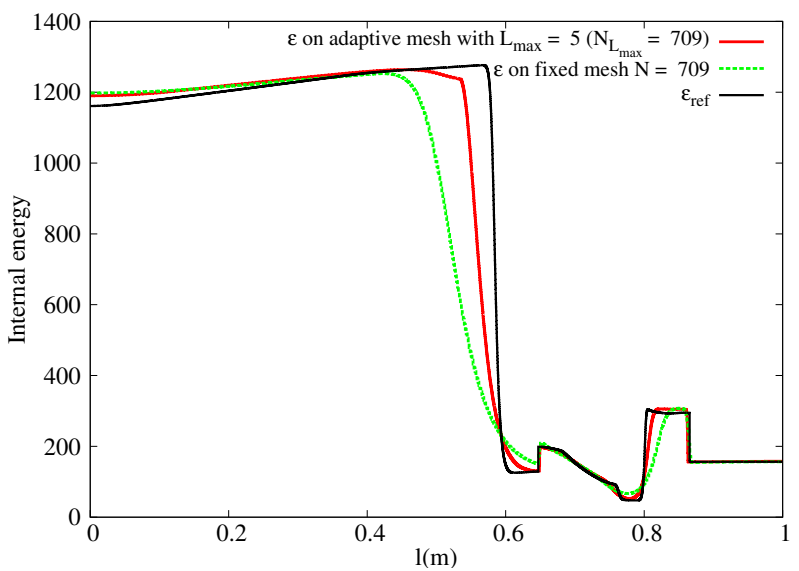

(d) Internal energy.

Figure 2: Comparison between AB1 (green lines), AB1U (red lines), and reference (black lines) cases for the density (a), the pressure (b), the momentum (c) and the internal energy (d) at time $t=0.038 \mathrm{~s}$ with $L_{\max }=5$. Numerical density of entropy production (in absolute value) is also plotted in subfigure (a).

Figure 3 plots, for both first (left) and second (right) order scheme, a mesh convergence study based on the evolution of $\left\|\varepsilon-\varepsilon_{e x}\right\|_{l_{x}^{1}}$ discretization error. As already noticed in [6], the rate of convergence is considerably increased by the adaptive scheme and, in our experiences, it can be improved by changing the threshold parameters $\alpha_{\min }$ and $\alpha_{\max }$ at the expense of the cpu-time. Finally, one notes the clear improvement of mesh convergence for both first and second-order schemes. In addition, we provide the numerical error for the density, pressure, velocity and energy for both first and second order scheme in (tab. (1))and (tab. (2)). 


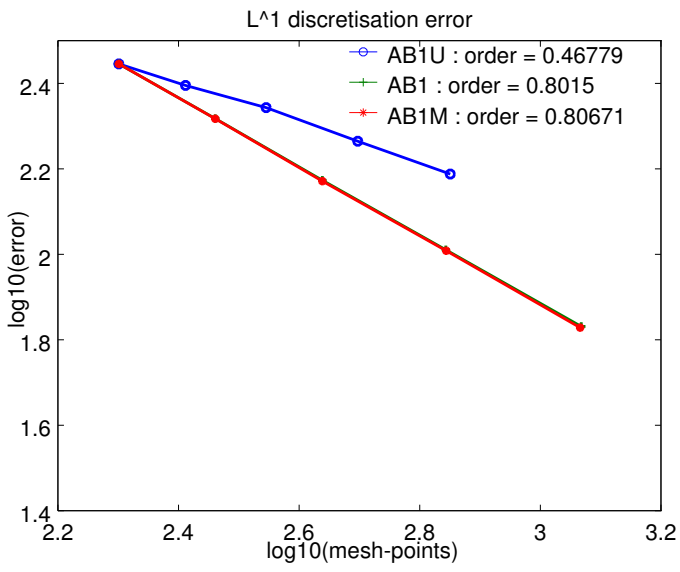

(a) First order scheme.

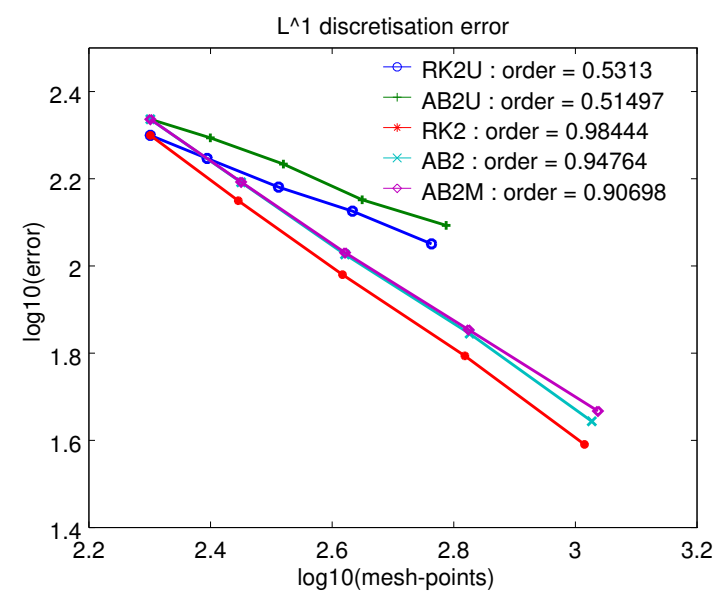

(b) Second order scheme.

Figure 3: $\left\|\varepsilon-\varepsilon_{e x}\right\|_{l_{x}^{1}}$ with respect to the average number of cells at time $t=0.038$.

\begin{tabular}{|c|c|c|c|c|c|c|c|}
\hline$L_{\max }$ & $\left\|\rho-\rho_{e x}\right\|_{l_{x}^{1}}$ & $\left\|p-p_{e x}\right\|_{l_{x}^{1}}$ & $\left\|u-u_{e x}\right\|_{l_{x}^{1}}$ & $\left\|\varepsilon-\varepsilon_{e x}\right\|_{l_{x}^{1}}$ & $N_{T_{f}}$ & $\mathrm{cpu}_{\mathrm{g}}$ & $\mathrm{cpu}_{1}$ \\
\hline \hline 1 & $0.645 \mathrm{E}+00$ & $0.259 \mathrm{E}+02$ & $0.104 \mathrm{E}+01$ & $0.279 \mathrm{E}+03$ & 200 & 1.30 & 1.30 \\
\hline 2 & $0.528 \mathrm{E}+00$ & $0.192 \mathrm{E}+02$ & $0.923 \mathrm{E}+00$ & $0.208 \mathrm{E}+03$ & 289 & 3.38 & 2.48 \\
\hline 3 & $0.409 \mathrm{E}+00$ & $0.141 \mathrm{E}+02$ & $0.801 \mathrm{E}+00$ & $0.149 \mathrm{E}+03$ & 435 & 9.16 & 6.21 \\
\hline 4 & $0.294 \mathrm{E}+00$ & $0.104 \mathrm{E}+02$ & $0.679 \mathrm{E}+00$ & $0.102 \mathrm{E}+03$ & 697 & 25.78 & 17.61 \\
\hline 5 & $0.197 \mathrm{E}+00$ & $0.796 \mathrm{E}+01$ & $0.588 \mathrm{E}+00$ & $0.678 \mathrm{E}+02$ & 1169 & 73.22 & 50.98 \\
\hline
\end{tabular}

Table 1: Convergence tests for first order AB1 scheme, $l_{x}^{1}$-norm at final time with respect to the averaged number of cells $N_{L_{\max }}$ of $\rho, u, p$ and $\varepsilon, \mathrm{cpu}_{\mathrm{g}}$ for global time stepping and $\mathrm{cpu}_{1}$ local time stepping, $N_{T_{f}}$ being the number of cells at the final time.

\begin{tabular}{|c|c|c|c|c|c|c|c|}
\hline$L_{\max }$ & $\left\|\rho-\rho_{e x}\right\|_{l_{x}^{1}}$ & $\left\|p-p_{e x}\right\|_{l_{x}^{1}}$ & $\left\|u-u_{e x}\right\|_{l_{x}^{1}}$ & $\left\|\varepsilon-\varepsilon_{e x}\right\|_{l_{x}^{1}}$ & $N_{T_{f}}$ & $\mathrm{cpu}_{\mathrm{g}}$ & $\mathrm{cpu}_{1}$ \\
\hline \hline 1 & $0.548 \mathrm{E}+00$ & $0.193 \mathrm{E}+02$ & $0.872 \mathrm{E}+00$ & $0.217 \mathrm{E}+03$ & 200 & 1.31 & 1.36 \\
\hline 2 & $0.421 \mathrm{E}+00$ & $0.132 \mathrm{E}+02$ & $0.706 \mathrm{E}+00$ & $0.156 \mathrm{E}+03$ & 282 & 3.32 & 2.46 \\
\hline 3 & $0.301 \mathrm{E}+00$ & $0.929 \mathrm{E}+01$ & $0.566 \mathrm{E}+00$ & $0.106 \mathrm{E}+03$ & 418 & 8.82 & 5.64 \\
\hline 4 & $0.202 \mathrm{E}+00$ & $0.672 \mathrm{E}+01$ & $0.448 \mathrm{E}+00$ & $0.700 \mathrm{E}+02$ & 670 & 23.64 & 15.26 \\
\hline 5 & $0.127 \mathrm{E}+00$ & $0.528 \mathrm{E}+01$ & $0.396 \mathrm{E}+00$ & $0.440 \mathrm{E}+02$ & 1064 & 65.01 & 41.86 \\
\hline
\end{tabular}

Table 2: Convergence tests for second order AB2 scheme, $l_{x}^{1}$-norm at final time with respect to the averaged number of cells $N_{L_{\max }}$ of $\rho, u, p$ and $\varepsilon, \mathrm{cpu}_{\mathrm{g}}$ for global time stepping and $\mathrm{cpu}_{1}$ local time stepping, $N_{T_{f}}$ being the number of cells at the final time. 


\begin{tabular}{|c|c|c|c|c|}
\hline Rate & $\left\|\rho-\rho_{e x}\right\|_{l_{x}^{1}}$ & $\left\|p-p_{e x}\right\|_{l_{x}^{1}}$ & $\left\|u-u_{e x}\right\|_{l_{x}^{1}}$ & $\left\|\varepsilon-\varepsilon_{e x}\right\|_{l_{x}^{1}}$ \\
\hline \hline AB1U & 0.35 & 0.60 & 0.41 & 0.47 \\
\hline AB1 & 0.67 & 0.67 & 0.33 & 0.80 \\
\hline AB1M & 0.66 & 0.68 & 0.35 & 0.81 \\
\hline RK2U & 0.44 & 0.70 & 0.55 & 0.53 \\
\hline AB2U & 0.43 & 0.65 & 0.42 & 0.51 \\
\hline RK2 & 0.90 & 0.75 & 0.48 & 0.98 \\
\hline AB2 & 0.87 & 0.77 & 0.48 & 0.95 \\
\hline AB2M & 0.77 & 0.72 & 0.39 & 0.91 \\
\hline
\end{tabular}

Table 3: Convergence tests for first and second order.

It is here demonstrated that the adaptive grid strategy using the numerical density of entropy production can considerably increase the accuracy without imposing a large number of cells and related extra CPU time required in solving the problem on a uniform grid.

\subsubsection{Two dimensional Riemann Problem}

Our tests are now extended to the two dimensional Riemann problem on the unit square with the following initial data

$$
(\rho, u, v, p)(0, x, y)= \begin{cases}(1,0,-0.4,1) & \text { if } x>0.5 \text { and } y>0.5 \\ (2,0,-0.3,1) & \text { if } x<0.5 \text { and } y>0.5 \\ (1.0625,0,0.2145,0.4) & \text { if } x<0.5 \text { and } y<0.5 \\ (0.5197,0,-1.1259,0.4) & \text { if } x>0.5 \text { and } y<0.5\end{cases}
$$

In this configuration, the solution consists of two contacts (North and South), a shock (West) and a rarefaction (east) as displayed in Fig. 4. A reference solution is computed on a uniform grid with 1000000 cells using the RK2U scheme. Figure 5 presents the reference solution at time $t=0.3$ with pressure (from 0.53 to 1.98 by 0.05 steps) and velocity field in subfigure 4, density in subfigure $5($ a) and numerical density of entropy production in subfigure 5(b). One notes that the shock and the contacts are associated with strong production of numerical density of entropy, which again demonstrates the relevancy of such numerical quantity to describe the most tricky regions of the computational domain.

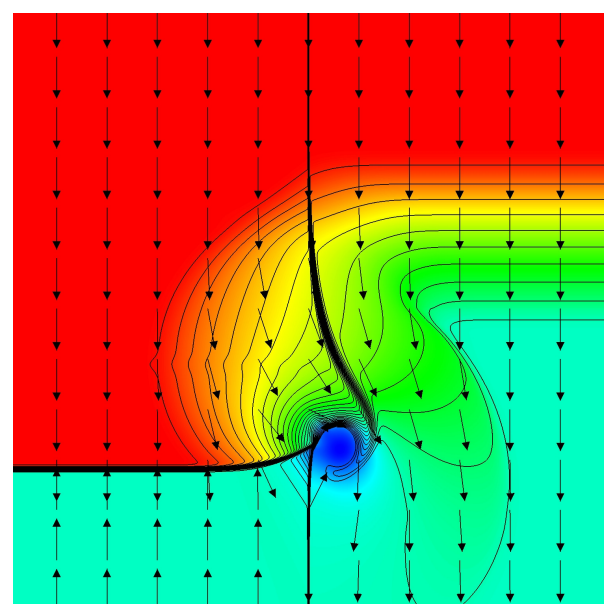

Figure 4: Reference solution for the 2D Riemann problem at time $t=0.3$ : pressure (colour), density (contours; 0.53 to 1.98 step 0.05 ), velocity field (arrows). 


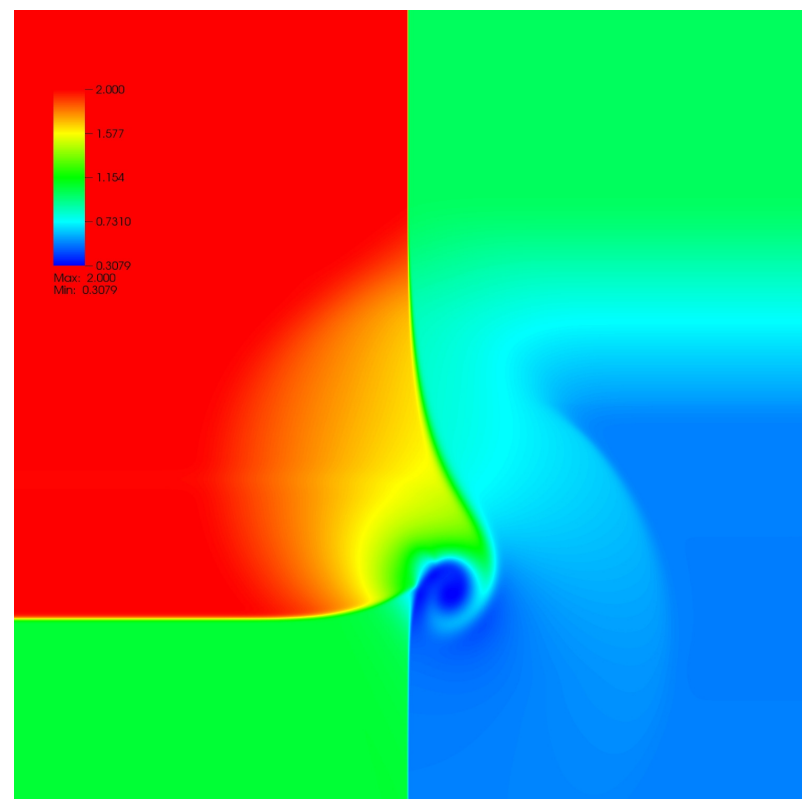

(a) Density (colour).

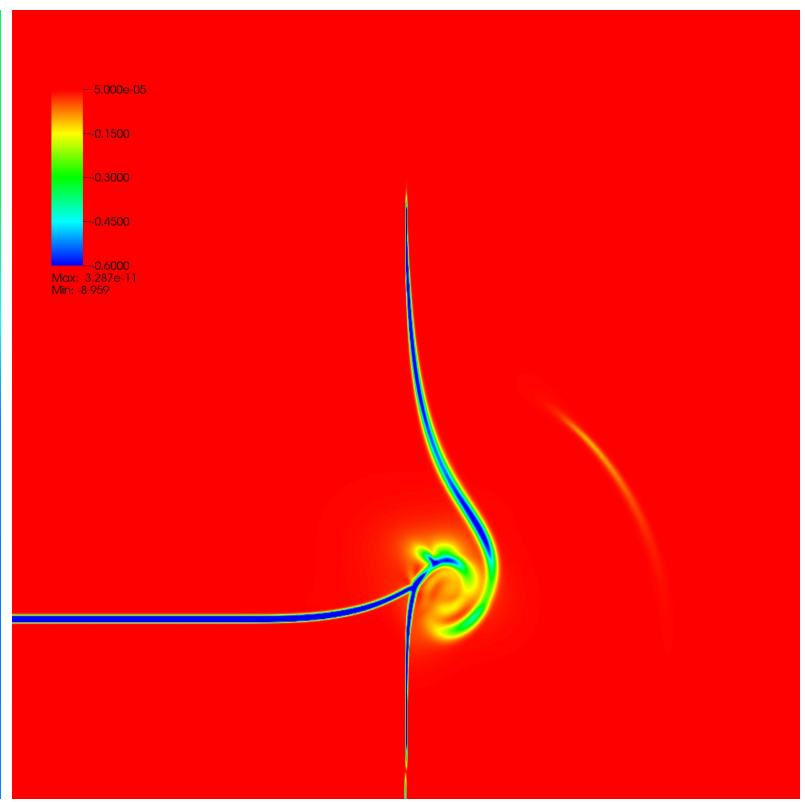

(b) Numerical density of entropy production.

Figure 5: Reference solution for the 2D Riemann problem at time $t=0.3$ and numerical density of entropy production.

For the following numerical experiments, we have used the following parameters:

CFL

Simulation time $(s)$

Initial number of blocks

$n_{x}=n_{y}$

Number of domain

Maximum level of mesh refinement

Mesh refinement parameter $\alpha_{\max }$

Mesh coarsening parameter $\alpha_{\min }$

Mesh refinement parameter $\bar{S}$

$$
\begin{array}{ll}
: & 0.5, \\
: & 0.3, \\
: & 30 \times 30, \\
: & 1 \\
: & 1, \\
: & L_{\max } . \\
: & 0.08, \\
: & 0.05 \\
: & \frac{1}{|\Omega|} \sum_{k_{b}} S_{k_{b}}^{n} .
\end{array}
$$

Convergence study are performed here with first order scheme only for AB1, AB1U and AB1M with the discrete $l_{x}^{1}$-norm for density and pressure.. Computational time are provided keeping in mind that model performance can be easily enhanced by the use of multi-domains as proposed in the next numerical experiments. The error between considered and reference solutions are performed by projecting the former on the fine grid of reference solution.

For the $\mathrm{AB} 1$ and $\mathrm{AB} 1 \mathrm{M}$ schemes, four tests have been carried out by varying the level of mesh refinement from 1 to 4 . As quoted before, the errors between the two schemes being similar, we only plot the point-wise pressure error between reference and AB1 case (see Fig. 6). Figure 7 shows pressure contours and mesh refinement. As expected, increasing the level of mesh refinement based on the numerical density of entropy production leads to a better description of the most difficult part of the problem: shock, rarefaction and contact regions, for instance. In comparison with other results, see for instance [22], the contact is precisely characterized, without any spurious vorticity and one observes the ripple formed in the NW quadrant. 


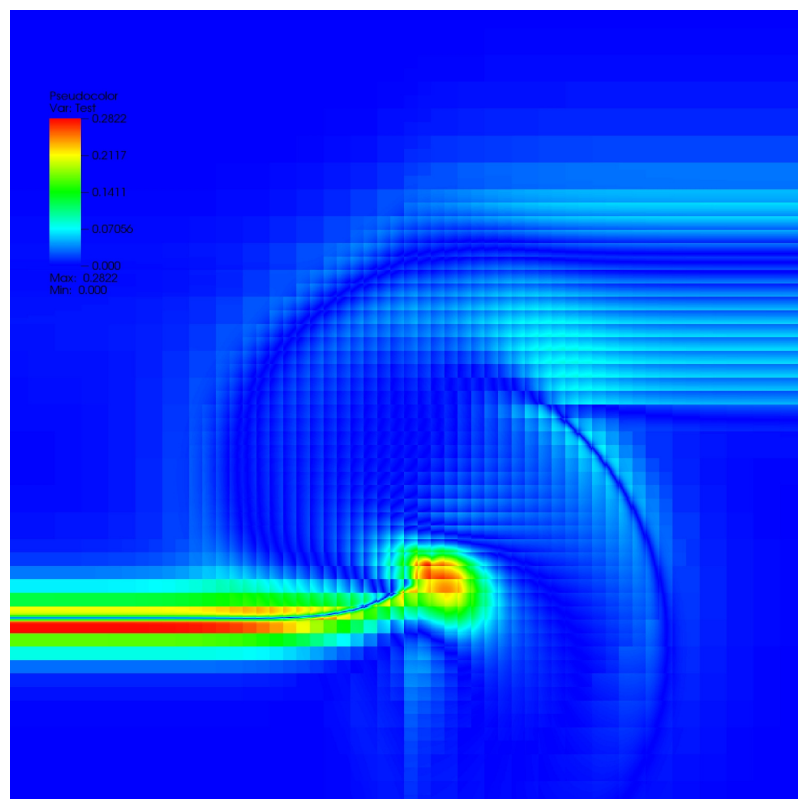

(a) $L_{\max }=1$.

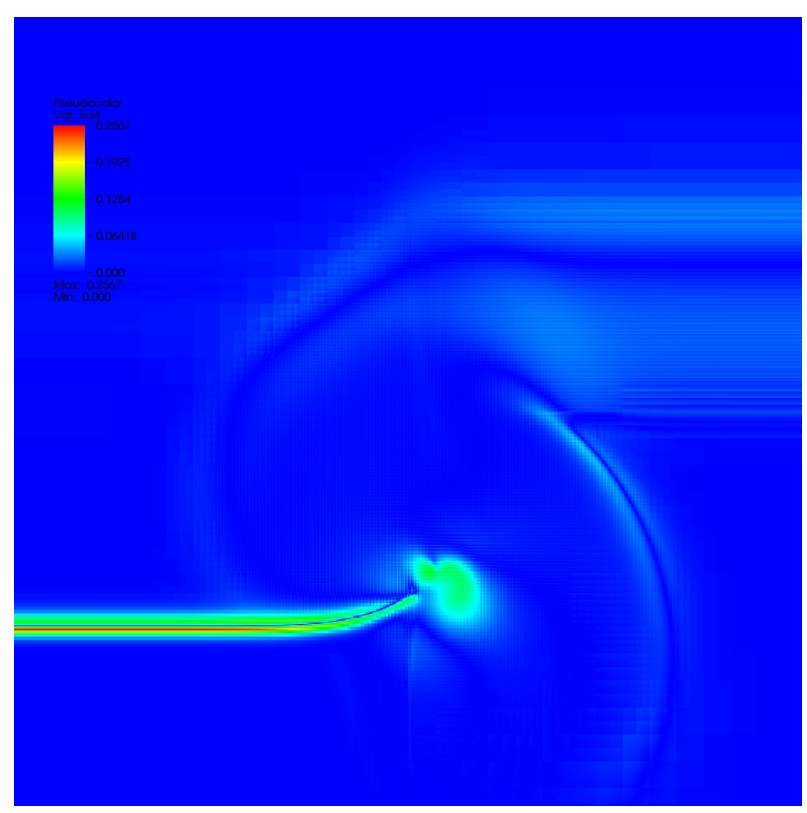

(c) $L_{\max }=3$.

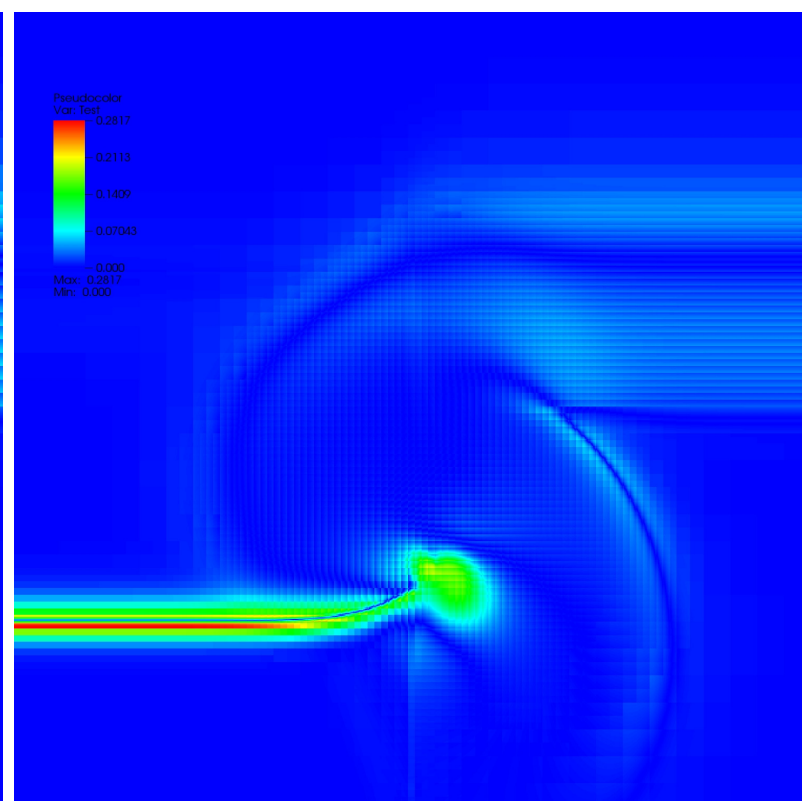

(b) $L_{\max }=2$.

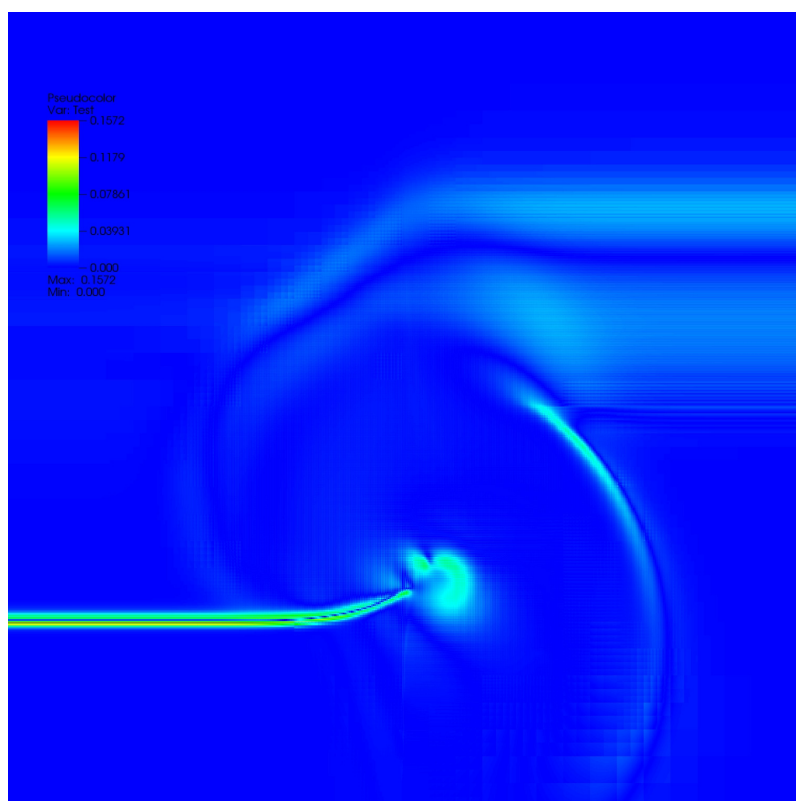

(d) $L_{\max }=4$.

Figure 6: Pointwise pressure error between the approximate solution and the reference one at time $t=0.3$. 


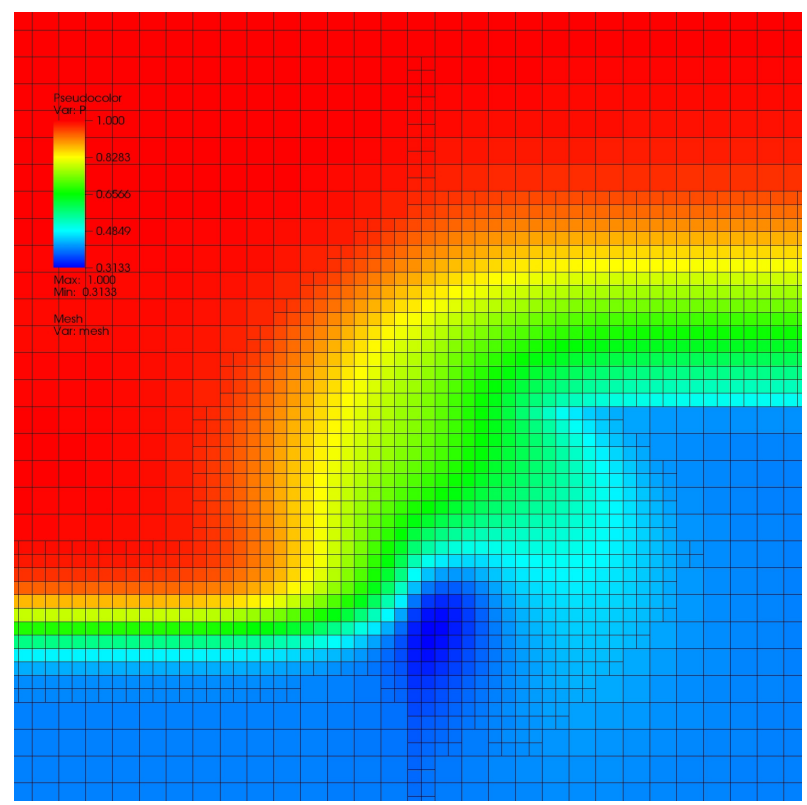

(a) $L_{\max }=1$.

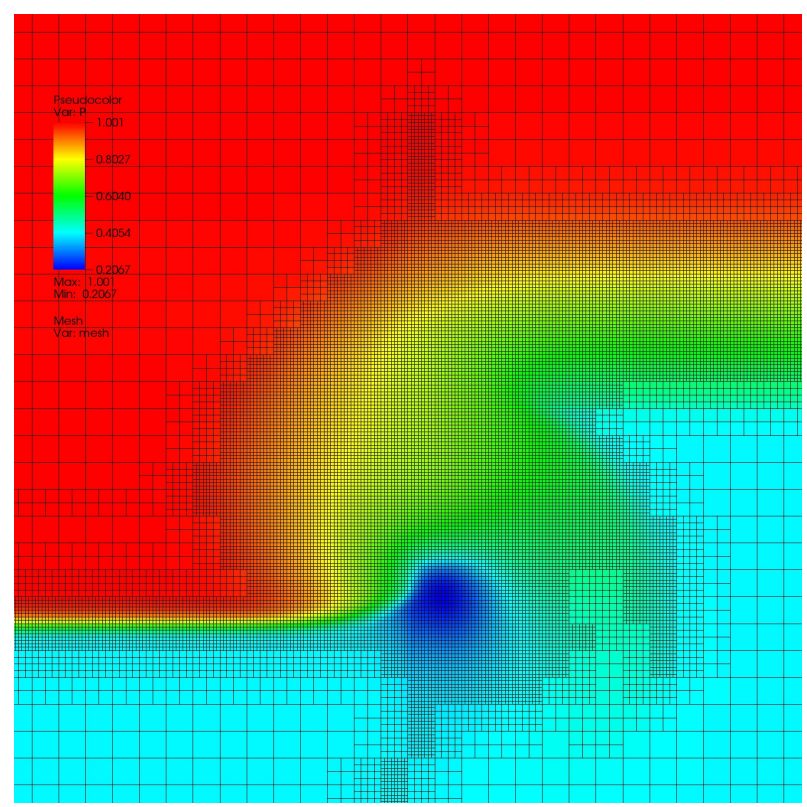

(c) $L_{\max }=3$.

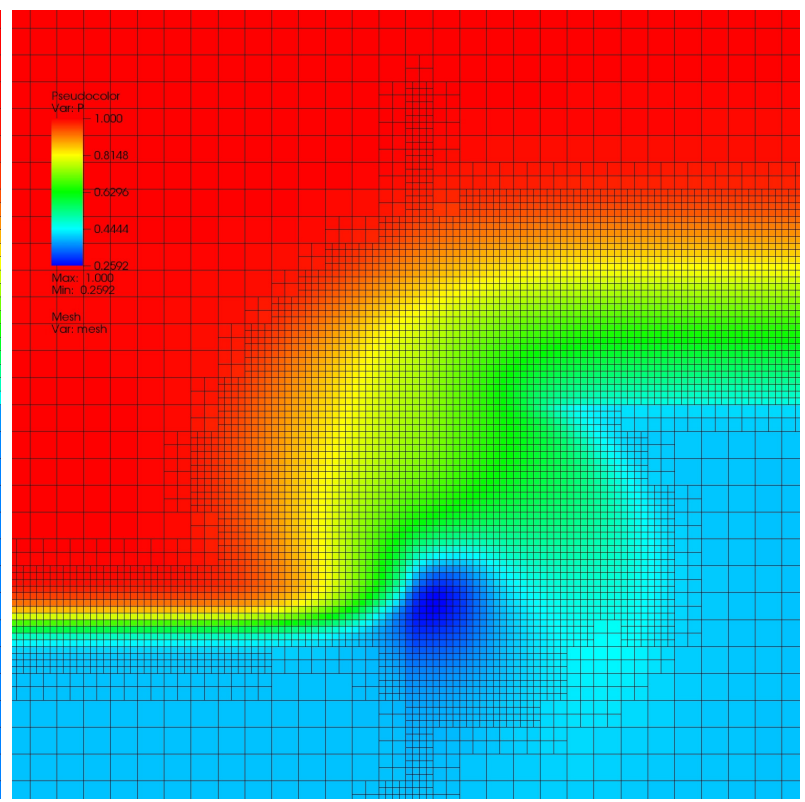

(b) $L_{\max }=2$.

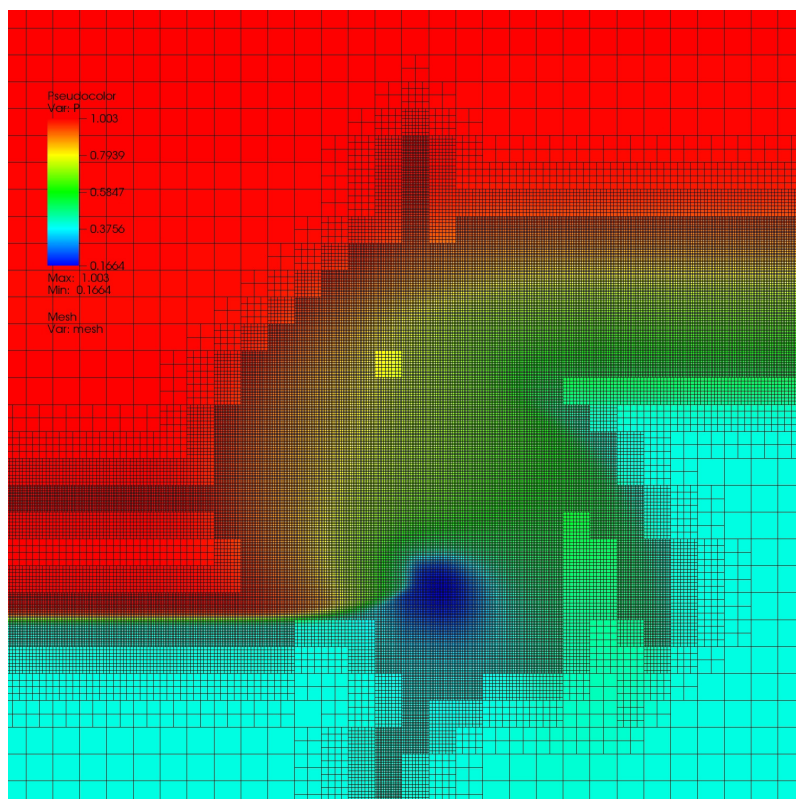

(d) $L_{\max }=4$.

Figure 7: Pressure and the mesh at time $t=0.3$.

Figures $8(\mathrm{a})$ and $8(\mathrm{~b})$ compare the errors with the discrete $l_{x}^{1}$-norm for density and pressure for the AB1, $\mathrm{AB} 1 \mathrm{M}$ and $\mathrm{AB} 1 \mathrm{U}$ schemes. As expected in the presence of contact discontinuity, the order of convergence is of order $1 / 2$ for all schemes. 


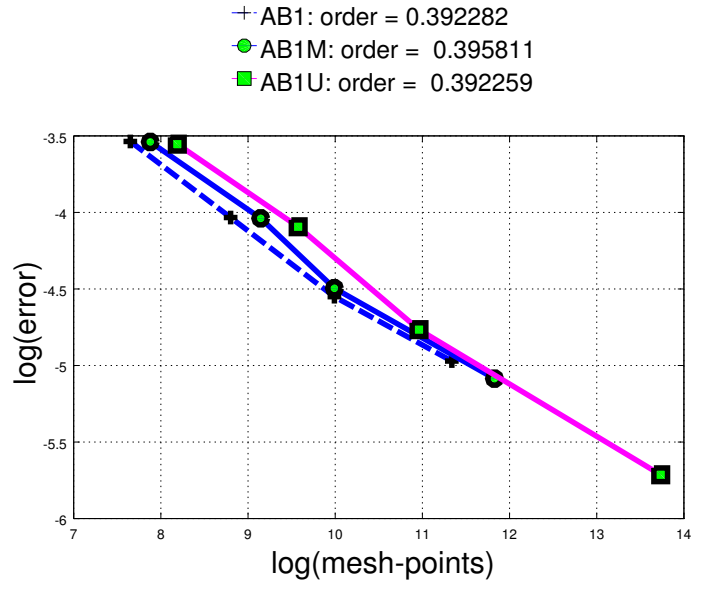

(a) Density.

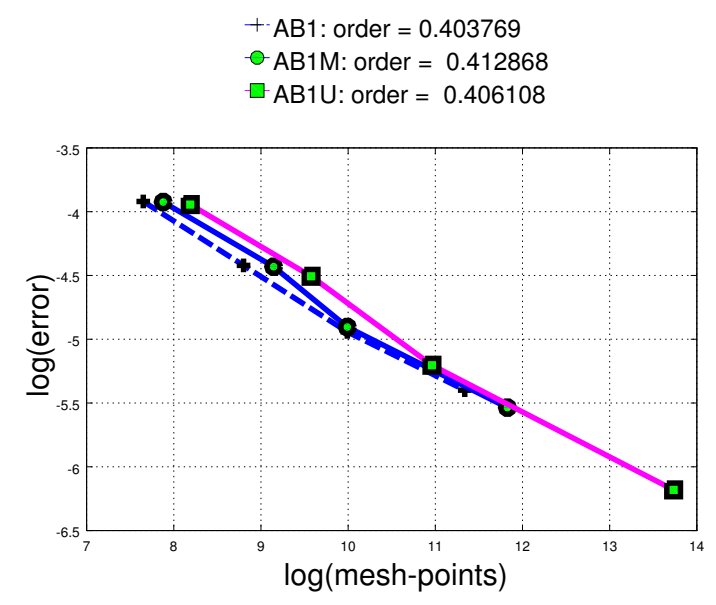

(b) Pressure.

Figure 8: Error analysis for the density and the pressure: number of cells vs $l_{x}^{1}$-error in log scale.

\subsection{Compressible low Mach two-phase flows equations and interface sharpening}

Let us consider now a compressible two-fluid flows problem where viscosity, surface tension and heat conduction are neglected. The incompressibility condition is relaxed using a low Mach approach in order to lead to an hyperbolic system of conservation laws. Thus, based on [11], the following three-dimensional isothermal hyperbolic and compressible Euler equations system is applied to a mixture fluid of air and water:

$$
\begin{cases}\frac{\partial \rho}{\partial t}+\nabla \cdot(\rho \boldsymbol{u}) & =0 \\ \frac{\partial(\rho \boldsymbol{u})}{\partial t}+\nabla \cdot(\rho \boldsymbol{u} \otimes \boldsymbol{u})+\nabla p & =\rho \boldsymbol{g}\end{cases}
$$

where the unknowns are the density $\rho$, the three components of the velocity $\boldsymbol{u}=(u, v, w)$, the pressure $p$. Here, $\boldsymbol{g}$ stands for gravitational acceleration.

Air and water fractions within the mixture are defined by the volume fraction function $\varphi \in[0,1](\varphi=0$ in the water, and $\varphi=1$ in the air). With this definition of $\varphi$, the pressure of the two-phase flow problem is a function of the density $\rho$ and the volume fraction $\varphi$, where $\varphi$ solves the following non-conservative transport equation:

$$
\frac{\partial \varphi}{\partial t}+\boldsymbol{u} \cdot \nabla \varphi=0
$$

It is usually admitted that a flow is incompressible if the Mach number $M=\|\boldsymbol{u}\| / c$ is lower than 0.1 ( $c$ is the sound speed), keeping in mind that the real (physical) Mach number is generally much smaller (of the order of $1 / 400 \sim 1 / 1600$ ). In particular, this is constraining for explicit finite volume solver in which the time step $\Delta t$ needs to satisfy a CFL (Courant-Friedrichs-Lewy) condition. Note also that the numerical scheme efficiency is expected to decrease with the Mach number. Therefore, an artificial pressure law (isothermal equation of state) is used to close the system :

$$
p=c_{0}^{2}\left(\rho-\left(\varphi \rho_{A}+(1-\varphi) \rho_{W}\right)\right)+p_{0} .
$$

In this expression, $\rho_{A}$ and $\rho_{W}$ stand for air and water densities, respectively, $c_{0}$ is the artificial sound speed (defined below) and $p_{0}$ a reference pressure. For further details about the EOS choice, the reader is referred to [11]. The value of $c_{0}$ is chosen as a compromise between the limits of compressible effects, the rate of numerical diffusion and a reasonable CFL constraint. In the present context, i.e. for flow velocity of the order 
of $1 \mathrm{~m} / \mathrm{s}$, an "optimized" value $c_{0}=20 \mathrm{~m} / \mathrm{s}$ is used. It is emphasized that in the boundary mixture region $0<\varphi<1$ related to numerical diffusion processes, the proposed pressure law has no physical meanings.

In the present case, for the two-fluid model, the expression of entropy and entropy flux in (2) are

$$
s=\frac{1}{2} \rho \boldsymbol{u}^{2}+c_{0}^{2} \rho \ln \rho-c_{0}^{2}\left(\rho_{W}-\rho_{A}\right) \varphi, \quad \psi=\left(\frac{1}{2} \rho \boldsymbol{u}^{2}+c_{0}^{2} \rho(\ln \rho+1)\right) \boldsymbol{u} .
$$

In the region $0<\varphi<1$ where both phases coexist, the numerical diffusion is expected to deteriorate the description of the air-water interface. Following [18, 28], an interface sharpening method is applied using a source term $S_{c}=\phi^{2}(1-\phi)^{2}(\phi-c)$ in the transport equation (9). The constant c is defined as a mass conservation parameter [15] leading to $\int_{\Omega} S_{c} \equiv 0$. For each time step, a fractional step method is used to solve eqs. (8). The non-homogeneous system is then solved with the interface sharpening source terms (with a 1-iteration explicit scheme):

$$
\begin{aligned}
& \frac{\partial \varphi}{\partial \tau}=S_{c} \\
& \frac{\partial \rho}{\partial \tau}=\frac{S_{c}}{\rho_{A}-\rho_{W}}, \\
& \frac{\partial \rho \boldsymbol{u}}{\partial \tau}=\frac{S_{c} \boldsymbol{u}}{\rho_{A}-\rho_{W}},
\end{aligned}
$$

where $\tau$ is a fictive time.

\subsubsection{A 2D dam-break problem}

The numerical model is confronted with the classical experiment of Martin and Moyce [24]. As shown in fig. 9, a column of water $(a \times 2 a)$ collapses in a box $(4 a \times 3 a)$. The initial mesh is composed of 594 blocks $(27 \times 22 \times 1)$ which are initially split into $n_{x} \times n_{y} \times n_{z}$ cells with $n_{x}=n_{y}=2$ and $n_{z}=1$. The mesh is refined around the air-water interface with a level 3 (i.e. 512 cells per block) with a total initial number of cells around 10500 cells (since the level between blocks cannot exceed 2). Blocks are then distributed on 10 domains which evolve during each re-meshing time step (see figure 11). Symmetry boundary conditions are imposed. The simulation time is $T=0.4 s$ and the AMR time step is fixed to $0.01 s$, i.e. the re-meshing occurs 40 times during the simulation with the mesh refinement and coarsening parameters respectively set to $\alpha_{\max }=0.2$ and $\alpha_{\min }=0.02$. During the global simulation, the number of cells evolves from 7500 to 17500 cells as shown in figure 14. Note that, even if the computation have been initially started with a very large number of cells, the mesh would have been quickly coarsened and automatically adapted to the production of the numerical density of entropy as displayed in figures 10 and 12 .

Figure 13 shows the rapid collapse of the water column and the impact on the right wall after $t=0.3 \mathrm{~s}$. The interface sharpening method together with the dynamic mesh refinement shown in fig. 10 thanks to the entropy production (fig. 12) allows an accurate description of the interface. A very good quantitative agreement in obtained with the experimental data of Martin and Moyce [24], as shown in fig. 15 in nondimensional data.

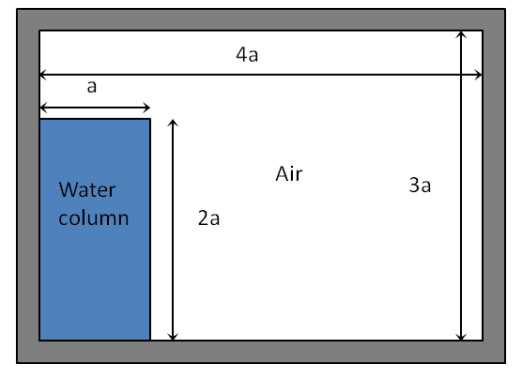

Figure 9: Collapse of water column [19, 24] 

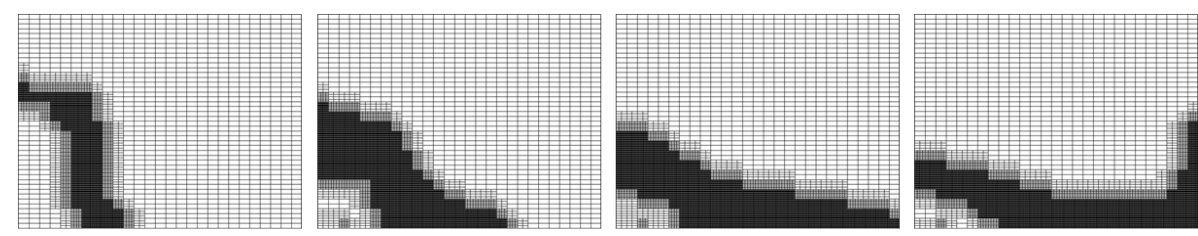

Figure 10: Mesh at time $0.1 \mathrm{~s}, 0.2 \mathrm{~s}, 0.3 \mathrm{~s}, 0.4 \mathrm{~s}$.
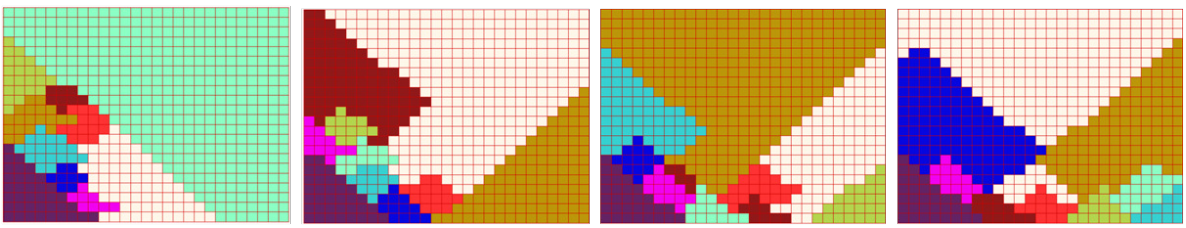

Figure 11: Block distribution in 10 domains at time $0.1 \mathrm{~s}, 0.2 \mathrm{~s}, 0.3 \mathrm{~s}, 0.4 \mathrm{~s}$.
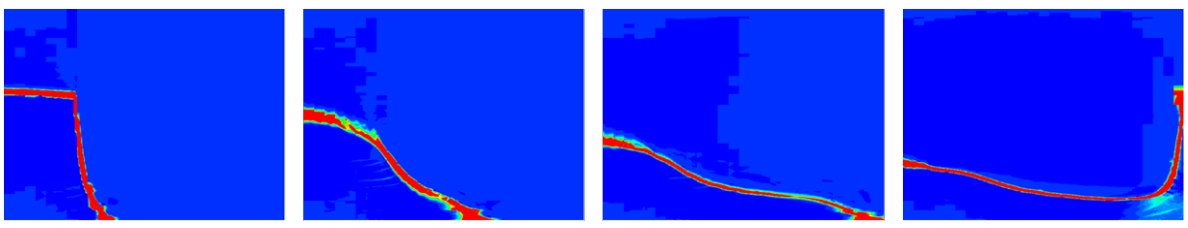

Figure 12: Numerical production of entropy at time $0.1 \mathrm{~s}, 0.2 \mathrm{~s}, 0.3 \mathrm{~s}, 0.4 \mathrm{~s}$ (blue zero, red negative values).
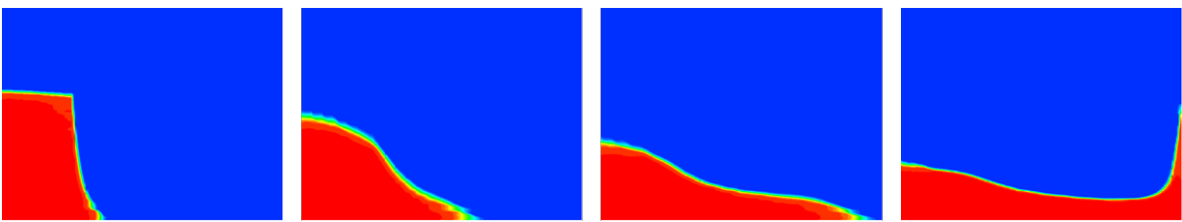

Figure 13: Density at time $0.1 \mathrm{~s}, 0.2 \mathrm{~s}, 0.3 \mathrm{~s}, 0.4 \mathrm{~s}$ (blue-air, red-water).

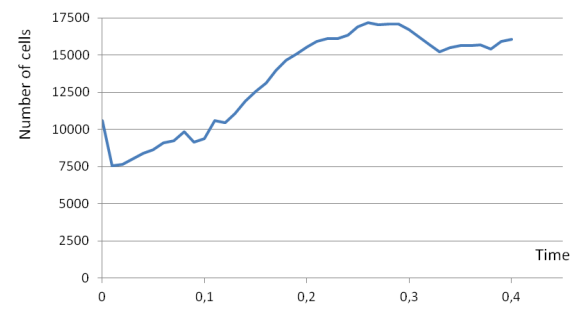

Figure 14: Number of cells during the computation. 


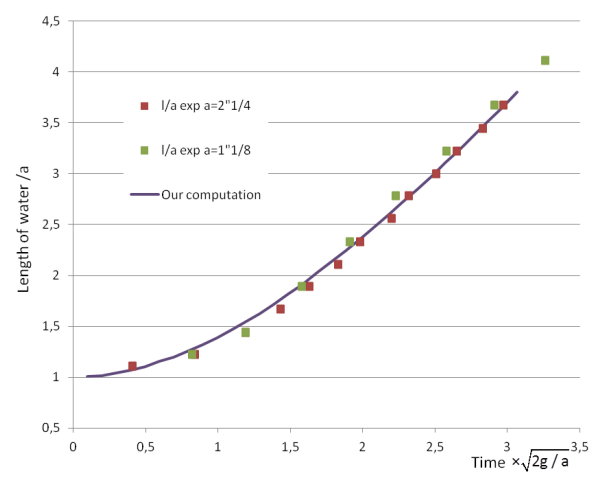

Figure 15: Length of Water during the collapsing. Confrontation between the computation and the experiment of Martin and Moyce [24].

\subsubsection{A 3D dam-break problem}

A 3D dam-break problem is here used as a benchmark test to evaluate the method speed-up potential in three dimensions.

We consider a water column $\left(\frac{1}{2} \times \frac{1}{2} \times \frac{1}{2}\right)$ collapsing in a unit cube. The initial mesh is composed of 3375 blocks $(15 \times 15 \times 15)$ which are initially split into $n_{x} \times n_{y} \times n_{z}$ cells with $n_{x}=n_{y}=n_{z}=1$. As done in previous test case, the blocks around the air-water interface are of level 3 . The simulation time is $\mathrm{T}=2.5$ and the AMR time step is fixed to 0.025, i.e. the re-meshing occurs 100 times during the simulation with the mesh refinement and coarsening parameters respectively set to $\alpha_{\max }=0.2$ and $\alpha_{\min }=0.02$. During the global simulation, the number of cells evolves from 172215 to 587763 . Snapshots of the simulation are shown in figure 16. The 3D dam-break collapse is well simulated, as well as the multiple reflection against the walls.

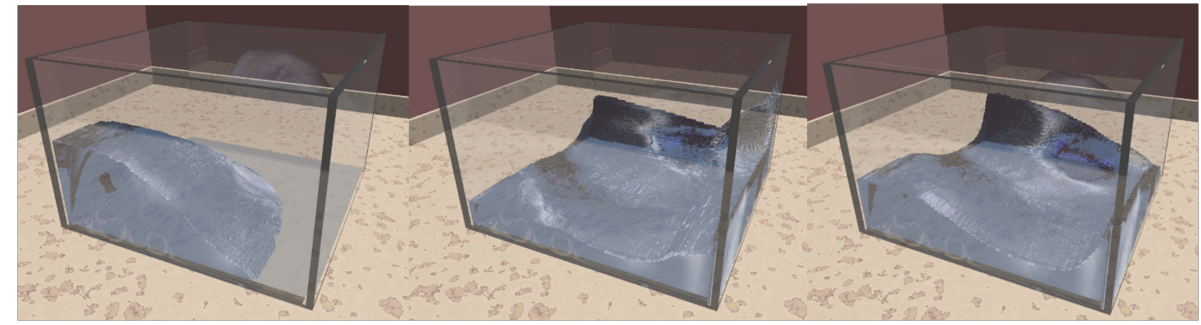

Figure 16: Collapse of $3 \mathrm{D}$ water column at time $t=0.25,0.50,0.75 \mathrm{~s}$

For the sake of comparison, each simulation has been computed on the same small cluster (2 nodes, 40 cores). In order to show the efficiency of the parallel implementation, the RK2 and AB2 CPU times are compared during the first AMR time step. The normalized cpu time, shown in fig. 17, stands for the inverse of the so-called speedup. The computational domain is split in 1, 2, 4, 8, 16 and 32 domains.

As expected, the Adams-Bashforth scheme with local time stepping allows a great improvement of the CPU time. The efficiency, i.e. $\frac{\text { speed up }}{\text { number of processors }}$, of the computation is roughly $85 \%$ for 8 domains and $60 \%$ for 32 domains. From a numerical point of view, the presented method is robust although the efficiency of the parallel process reaches a steady state after 20 processors, indicating that the parallel procedure has to be optimized. 


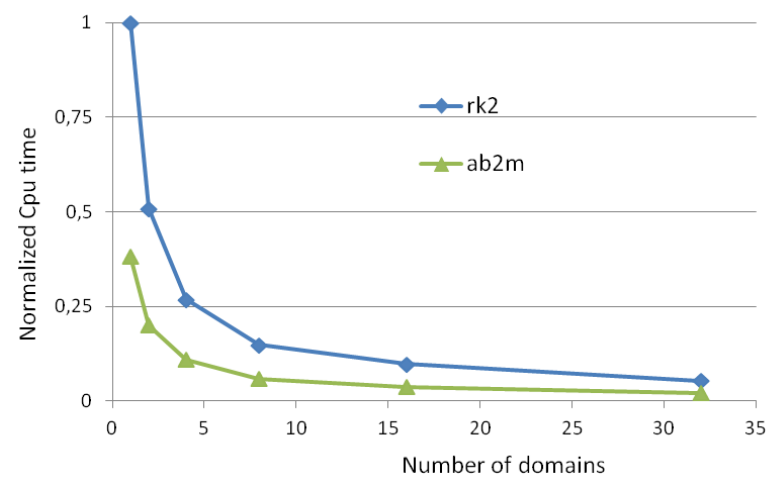

Figure 17: Normalized cpu time versus number of domains with Runge-Kutta or Adams-Bashforth scheme

\section{Conclusion}

This paper reports on the extension of the one dimensional scheme presented by Ersoy et al. [6] to a general multidimensional framework. A finite volume solver is used to solve the non-linear hyperbolic equation system on unstructured meshes. An Adaptative Mesh Refinement is introduced to improve both solution accuracy and cpu performance. It is based on a useful numerical criterion: the numerical density of entropy production. From a computational viewpoint, to make the local time step method more efficient in a parallel context, a new Block-Based AMR technique (BB-AMR) is applied.

A series of 1D, 2D and 3D test cases have been performed to test and validate our approach, using several model configurations (uniform or adaptative grids, Adams-Bashforth or Runge-Kutta schemes, etc). 1D and 2D Riemann problems demonstrated that the adaptative grid strategy using the numerical density of entropy production helps to greatly improve the accuracy and reduce the computational effort. Dambreak benchmarks have also been carried out, both in 2 and $3 \mathrm{D}$, to test our method when applied to compressible low Mach two-phase flows problems. Interface sharpening techniques are used here to improve the description of the complex wavy motion of the free surface during the collapse of water columns and impacts on rigid walls. Very good agreement is obtained with existing experimental results. Comparison between AdamsBashforth and Runge-Kutta shows the significant computation speed-up provided by the former scheme.

Further on-going developments concern in particular the optimization of the proposed numerical scheme in order to increase the efficiency (i.e. the ratio of the speedup over the number of process) as pointed out in the last three-dimensional test case.

\section{Acknowledgements}

This work is supported by the ModTerCom project within the APEX program of the region Provence-AlpeCôte d'Azur.

\section{References}

[1] T. Allahviranloo, N. Ahmady, And E. Ahmady, Numerical solution of fuzzy differential equations by predictor-corrector method, Inform. Sci., 177 (2007), pp. 1633-1647.

[2] C. Altmann, T. Belat, M. Gutnic, P. Helluy, H. Mathis, E. Sonnendrücker, W. Angulo, AND J. HÉRARD, A local time-stepping discontinuous Galerkin algorithm for the MHD system, in CEM- 
RACS 2008-Modelling and numerical simulation of complex fluids, vol. 28 of ESAIM Proc., EDP Sci., Les Ulis, 2009, pp. 33-54.

[3] M. Berger And P. Colella, Local adaptive mesh refinement for shock hydrodynamics, Journal of Computational Physics, 82 (1989), pp. $64-84$.

[4] M. Berger and J. Oliger, Adaptive mesh refinement for hyperbolic partial differential equations, J. Comp. Phys., 53 (1984), pp. 484-512.

[5] T. Coupez And E. Hachem, Solution of high-reynolds incompressible flow with stabilized finite element and adaptive anisotropic meshing, Computer Methods in Applied Mechanics and Engineering, 267 (2013), pp. $65-85$.

[6] M. Ersoy, F. Golay, and L. Yushchenko, Adaptive multiscale scheme based on numerical density of entropy production for conservation laws, Cent. Eur. J. Math., 11 (2013), pp. 1392-1415.

[7] R. Eymard, G. T., And R. Herbin, Finite volume methods, in Handbook of numerical analysis, Vol. VII, Handb. Numer. Anal., VII, North-Holland, Amsterdam, 2000, pp. 713-1020.

[8] D. Fuster, G. Agbaglah, C. Josserand, S. Popinet, and S. Zaleski, Numerical simulation of droplets, bubbles and waves: state of the art, Fluid Dynamics Research, 41 (2009), p. 065001.

[9] E. Godlewski and P. Raviart, Numerical approximation of hyperbolic systems of conservation laws, vol. 118 of Applied Mathematical Sciences, Springer-Verlag, New York, 1996.

[10] F. Golay, M. Ersoy, L. Yushchenko, and D. Sous, Block-based adaptive mesh refinement scheme using numerical density of entropy production for three-dimensional two-fluid flows, International Journal of Computational Fluid Dynamics, 29 (2015), pp. 67-81.

[11] F. Golay and P. Helluy, Numerical schemes for low mach wave breaking, International Journal of Computational Fluid Dynamics, 21(2) (2007), pp. 69-86.

[12] E. Hachem, S. Feghali, R. Codina, and T. Coupez, Immersed stress method for fluid structure interaction using anisotropic mesh adaptation, International Journal for Numerical Methods in Engineering, 94 (2013), pp. 805-825.

[13] E. Hairer, S. P. Nørsett, And G. Wanner, Solving ordinary differential equations. I, vol. 8 of Springer Series in Computational Mathematics, Springer-Verlag, Berlin, second ed., 1993.

[14] P. Houston, J. Mackenzie, E. Süli, And G. Warnecke, A posteriori error analysis for numerical approximations of Friedrichs systems, Numer. Math., 82 (1999), pp. 433-470.

[15] A. KaČEniauskas, Development of efficient interface sharpening procedure for viscous incompressible flows, Informatica, 19 (2008), pp. 487-504.

[16] S. KaRni AND A. KURGAnOv, Local error analysis for approximate solutions of hyperbolic conservation laws, Adv. Comput. Math., 22 (2005), pp. 79-99.

[17] S. Karni, A. Kurganov, and G. Petrova, A smoothness indicator for adaptive algorithms for hyperbolic systems, J. Comp. Phys., 178 (2002), pp. 323-341.

[18] S. KoKH, Aspects numériques et théoriques de la modélisation des écoulements diphasiques compressibles par des méthodes de capture d'interface, PhD thesis, Université de Paris VI, 2001.

[19] S. Koshizuka, H. Tamako, and Y. OKa, A particle method for incompressible viscous flow with fluid fragmentations, Computational Fluid Dynamics Journal, 4 (1995), pp. 29-46. 
[20] A. Kurganov and E. Tadmor, Solution of two-dimensional riemann problems for gas dynamics without riemann problem solvers, Numerical Methods for Partial Differential Equations, 18 (2002), pp. 584-608.

[21] P. D. LaX And X.-D. LiU, Solution of two-dimensional riemann problems of gas dynamics by positive schemes, SIAM Journal on Scientific Computing, 19 (1998), pp. 319-340.

[22] R. Liska And B. Wendroff, Comparison of several difference schemes on 1d and 2d test problems for the euler equations, SIAM Journal on Scientific Computing, 25 (2003), pp. 995-1017.

[23] F. Losasso, F. Gibou, And R. Fedkiw, Simulating water and smoke with an octree data structure, ACM Trans. Graph., 23 (2004), pp. 457-462.

[24] J. Martin And W. Moyce, Part iv. an experimental study of the collapse of liquid columns on a rigid horizontal plane, Philosophical Transactions of the Royal Society of London. Series A, Mathematical and Physical Sciences, 244 (1952), pp. 312-324.

[25] C. Min And F. Gibou, A second order accurate level set method on non-graded adaptive cartesian grids, Journal of Computational Physics, 225 (2007), pp. $300-321$.

[26] A. N. Sambe, D. Sous, F. Golay, P. Fraunié, and R. Marcer, Numerical wave breaking with macro-roughness, European Journal of Mechanics - B/Fluids, 30 (2011), pp. 577 - 588.

[27] C. W. Schulz-Rinne, J. P. Collins, and H. M. Glaz, Numerical solution of the riemann problem for two-dimensional gas dynamics, SIAM Journal on Scientific Computing, 14 (1993), pp. 1394-1414.

[28] K.-M. ShyuE, An eulerian interface-sharpening algorithm for compressible gas dynamics, in Modeling, Simulation and Optimization of Complex Processes-HPSC 2012, Springer, 2014, pp. 221-231.

[29] E. F. Toro, Riemann solvers and numerical methods for fluid dynamics, Springer-Verlag, Berlin, third ed., 2009.

[30] M. J. Williamschen and C. Groth, Parallel anisotropic block-based adaptive mesh refinement algorithm for three-dimensional flows, in 21st AIAA Computational Fluid Dynamics Conference, June 24-27, San Diego, CA, 2013, pp. 1-22.

[31] P. Woodward AND P. COlELla, The numerical simulation of two-dimensional fluid flow with strong shocks, J. Comput. Phys., 54 (1984), pp. 115-173.

[32] P. R. Woodward, Trade-offs in designing explicit hydrodynamical schemes for vector computers, in Parallel computations, vol. 1 of Comput. Tech., Academic Press, Orlando, FL, 1982, pp. 153-171.

[33] K. Yiu, D. Greaves, S. Cruz, A. Saalehi, and A. Borthwick, Quadtree grid generation: Information handling, boundary fitting and cfd applications, Computers \& Fluids, 25 (1996), pp. 759 769.

[34] M. Zhang And W. Wu, A two dimensional hydrodynamic and sediment transport model for dam break based on finite volume method with quadtree grid, Applied Ocean Research, 33 (2011), pp. 297 - 308.

[35] T. Zhang And Y. X. Zheng, Conjecture on the structure of solutions of the riemann problem for twodimensional gas dynamics systems, SIAM Journal on Mathematical Analysis, 21 (1990), pp. 593-630.

[36] Z. Zheng And C. GROTh, Block-based adaptive mesh refinement finite-volume scheme for hybrid multiblock meshes, in 7st conference on Computational Fluid Dynamics (ICCFD7), July 9-13, Hawaii, 2012, pp. 1-19. 